\title{
Study Protocol Confidentiality Status
}

National Cancer Institute

\section{Source}

National Cancer Institute. Study Protocol Confidentiality Status. NCI Thesaurus. Code C71109.

A characteristic that defines how the disclosure of protocol content is controlled. 